\title{
Correction to: Nuclear Suitors or Would-be Proliferators
}

\section{Correction to: \\ P. F. Ikonomou, Global Nuclear Developments, https://doi.org/10.1007/978-3-030-46997-9_5}

This book was inadvertently published with missing citation number in Chapter 5 and other textual errors.

An entry had been missing in the citation number. Citation [43] was added and citations were renumbered throughout the Chapter; other textual corrections have also been made throughout the book. These have been corrected in the book.

The following corrections were made in Chapter 5.

On Chapter 5, Page 69, citation number was changed from [2] to [3]; on Page 70, citation numbers were changed from [2] to [4] and [3] to [5]; on Page 73, citation numbers were changed from [4] to [6], [5] to [7], [6] to [2], [7] to [8]; and Pages 74-90, all citations renumbered by 1, i.e., [8] to [9], [9] to [10] ... [42] to [43]. 